\title{
Structured LDPC Codes for High-Density Recording: Large Girth and Low Error Floor
}

\author{
J. Lu and J. M. F. Moura \\ Department of Electrical and Computer Engineering, Carnegie Mellon University, Pittsburgh PA 15213-3890 USA
}

\begin{abstract}
High-rate low-density parity-check (LDPC) codes are the focus of intense research in magnetic recording because, when decoded by the iterative sum-product algorithm, they show decoding performance close to the Shannon capacity. However, cycles, especially short cycles, are harmful to LDPC codes. The paper describes the partition-and-shift LDPC (PS-LDPC) codes, a new class of regular, structured LDPC codes that can be designed with large girth and arbitrary large minimum distance. Large girth leads to more efficient iterative decoding and codes with better error-floor properties than random LDPC codes. PS-LDPC codes can be designed for any desired column weight and with flexible code rates. The paper details the girth and distance properties of the codes and their systematic construction and presents analytical and simulation performance results that show that, in the high signal-to-noise ratio region, PS-LDPC codes outperform random codes, alleviating the error floor phenomenon.
\end{abstract}

Index Terms-Girth, LDPC code.

\section{INTRODUCTION}

$\mathbf{L}$ OW-DENSITY PARITY-CHECK (LDPC) codes [1] can be described by their corresponding Tanner graphs [2]. The length of the shortest cycle in a Tanner graph is referred to as girth $g$ of the graph. Tanner graphs with large girth are particularly desirable due to the following two reasons. In LDPC decoding, the sum-product algorithm yields its optimal a posteriori probability solution only for the first $\lfloor(g-2) / 4\rfloor$ iterations [1]. In successive iterations, short cycles in the Tanner graph tax the computing effort of the decoding algorithm and prevent it from converging to the optimal decoding result [3]. Hence, the larger the girth $g$, the more the optimal decoding iterations. A second reason for designing LDPC codes with large girth relates to the minimum distance of the code. Tanner [2] derives a lower bound on the minimum distance $d_{\min }$; this lower bound increases exponentially with the girth $g$ of the code. Therefore, LDPC codes with large girth imply good codes.

Among different types of LDPC codes, cyclic and quasi-cyclic LDPC codes are promising in applications since they have low-complexity encoding and decoding algorithms and simplify hardware implementation. Typical work includes finite-geometry codes [4], BIBD codes [5], algebraic construction [6], Fossorier's work [7], etc. For more literature on cyclic and quasi-cyclic codes, refer to [8], [9]. However, Fossorier [7] proved that the girth of cyclic and quasi-cyclic codes whose parity-check matrix has no zero blocks is at most 12 . Hence, the girth $g$ of such codes will not follow the log relationship $g \propto \log _{(j-1)(k-1)} n,[1]$, where $j$ is the column weight, $k$ is the row weight, and $n$ is the code block length. By the above analysis, cyclic and quasi-cyclic codes perform poorly at very long code block lengths. Their small girths limit their applications when very long codes are desired.

As far as we know, designing regular structured LDPC codes with arbitrary large girth and flexible code rates is still an interesting problem not yet solved. We solve this problem

Digital Object Identifier 10.1109/TMAG.2005.861748 by proposing a novel type of structured LDPC codes-partition-and-shift LDPC (PS-LDPC) codes that facilitates the systematic design of LDPC codes with arbitrary large girth, arbitrary column weight, and flexible code rates.

\section{LDPC CODES: PARTITION-AND-ShIFT DESIGNS}

We construct an LDPC code from its Tanner graph. Let $V_{c}$ be the set containing all $m$ check nodes and $V_{b}$ the set containing all $n$ bit nodes. Partition $V_{c}$ into $N_{c}$ disjoint subsets of the same size $p$ provided that $m=N_{c} \cdot p, p \in \mathbb{N}$. Similarly, partition $V_{b}$ into $N_{b}$ disjoint subsets of the same size $p$ provided that $n=$ $N_{b} \cdot p, p \in \mathbb{N}$. We require that $N_{c} \geq j$ and $N_{b} \geq k$ where $j$ and $k$ are the degrees of the bit nodes and check nodes, respectively. Elements in each subset are indexed from 0 to $p-1$. We also index the subsets in $V_{c}$ from 1 to $N_{c}$ and the subsets in $V_{b}$ from 1 to $N_{b}$. We name the parameter $p$ the cardinality of each subset.

We say a check node subset $\mathcal{A}$ is connected to a bit node subset $\mathcal{B}$ if:

1) each of the $p$ check nodes in $\mathcal{A}$ is connected to a bit node in $\mathcal{B}$;

2) different check nodes in $\mathcal{A}$ are connected to different bit nodes in $\mathcal{B}$.

Hence, the connection between two subsets sets up a one to one relationship between nodes of the two subsets. To construct a regular LDPC code with uniform check node degree $k$ and bit node degree $j$, we let each check node subset connect to $k$ bit node subsets and each bit node subset connect to $j$ check node subsets. We further enforce the following constraint: Every check node, indexed by $X_{c}$ in the $\alpha$ th check node subset is connected to the bit node indexed by $X_{b}$ in the $\beta$ th bit node subset, where $X_{b}=X_{c} \stackrel{p}{\oplus} S_{\alpha, \beta}$ where $0 \leq S_{\alpha, \beta} \leq p-1$ and $\stackrel{p}{\oplus}$ represents modulo- $p$ addition. We call LDPC codes defined in this way partition-and-shift LDPC (PS-LDPC) codes. Fig. 1 shows the Tanner graph for a PS-LDPC code. There are six subsets of bit nodes that a check node can be connected to but each check node is connected to only three of them.

The parameters $S_{\alpha, \beta}, 1 \leq \alpha \leq N_{c}, 1 \leq \beta \leq N_{b}$, in the above definition are called shifts. Check nodes in the same subset $\alpha$ of check nodes are connected to bit nodes in the same 


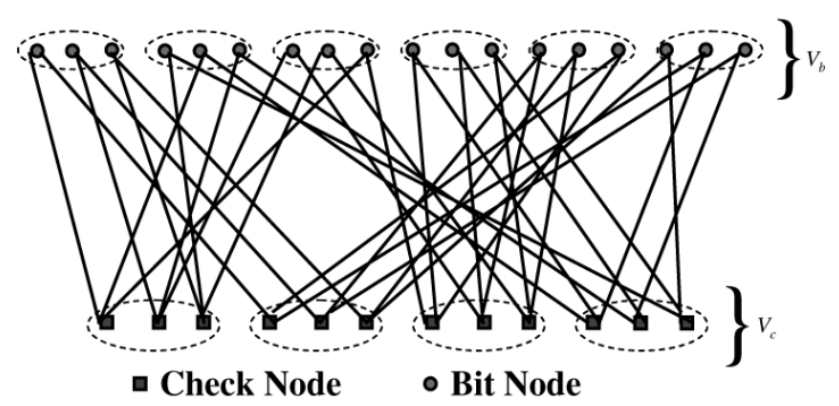

Fig. 1. Tanner graph for a PS-LDPC code.

subset $\beta$ of bit nodes by using the same shift $S_{\alpha, \beta}$. We collect all the shifts $S_{\alpha, \beta}$ in an $N_{c} \times N_{b}$ matrix called the shift matrix $\mathbf{S}=\left[\mathbf{S}_{\alpha, \beta}\right]$. The $\alpha$ th row of $\mathbf{S}$ stores those shifts associated with the $\alpha$ th subset of check nodes; the $\beta$ th column of $\mathbf{S}$ stores those shifts associated with the $\beta$ th subset of bit nodes. Hence, the entry in row $\alpha$ and column $\beta$ in $\mathbf{S}$ is the shift $S_{\alpha, \beta}$ that determines how to connect the $\alpha$ th check node subset to the $\beta$ th bit node subset. If there is no connection between the $\alpha$ th check node subset and the $\beta$ th bit node subset, we represent the corresponding entry by " $*$ ” on the shift matrix S. Since each check node subset is only connected to $k$ bit node subsets and there are totally $N_{c}$ check node subsets, there exist only $k \cdot N_{c}$ non-“*” shifts. For example, (1) shows the shift matrix $\mathbf{S}$ that corresponds to the Tanner graph shown in Fig. 1:

$$
\mathbf{S}=\left[\begin{array}{llllll}
0 & 1 & 2 & * & * & * \\
0 & * & * & * & 2 & 1 \\
* & * & 2 & 0 & 2 & * \\
* & 1 & * & 0 & * & 1
\end{array}\right]
$$

Code rate By the above construction, there are no limitations on the column weight $j$ and row weight $k$ of a PS-LDPC code. We can design PS-LDPC codes with arbitrary column weight $j$ and row weight $k$. For a regular code, the code rate $\rho=1-(j / k)$ if we omit a few linearly dependent parity-check equations. Therefore, we can generate PS-LDPC codes with any desired code rate by tuning the values of $j$ and $k$. for example, if a $\rho=8 / 9$ and column-weight- 3 PS-LDPC code is desired, set $j=3$ and $k=27$.

\section{PS-LDPC CODES: CYCLES AND SHIFTS}

Since our goal is to eliminate short cycles that are harmful to LDPC decoding, we first relate cycles in the Tanner graphs of PS-LDPC codes to closed paths in the shift matrix $\mathbf{S}$.

Theorem 1 (2t-CYCLE): The Tanner graph for a PS-LDPC code does NOT contain $2 t$-cycles if and only if any closed path of length $2 t$ in the shift matrix $\mathbf{S}$ with $2 t$ corners $S_{\alpha_{1}, \beta_{1}} \ldots S_{\alpha_{2 t}, \beta_{2 t}}$ violates the Cycle Condition $\oplus_{i=1}^{2 t}(-1)^{i+1} S_{\alpha_{i}, \beta_{i}}=S_{\alpha_{1}, \beta_{1}} \stackrel{p}{\ominus} S_{\alpha_{2}, \beta_{2}} \cdots \stackrel{p}{\oplus} S_{\alpha_{2 i-1}, \beta_{2 i-1}} \stackrel{p}{\ominus}$ $S_{\alpha_{2 i}, \beta_{2 i}} \ldots \stackrel{p}{\oplus} S_{\alpha_{2 t-1}, \beta_{2 t-1}} \stackrel{p}{\ominus} S_{\alpha_{2 t}, \beta_{2 t}}=0$ ( $\stackrel{p}{\ominus}$ represents modulo- $p$ subtraction).

Proof: if: We prove an equivalent proposition of the if part: If there exists a $2 t$-cycle in the Tanner graph, there must exist a closed path in the corresponding shift matrix $\mathbf{S}$ whose $2 t$ corners $S_{\alpha_{1}, \beta_{1}}, S_{\alpha_{2}, \beta_{2}}, \ldots, S_{\alpha_{2 t}, \beta_{2 t}}$ satisfy the Cycle Condition in the Theorem.
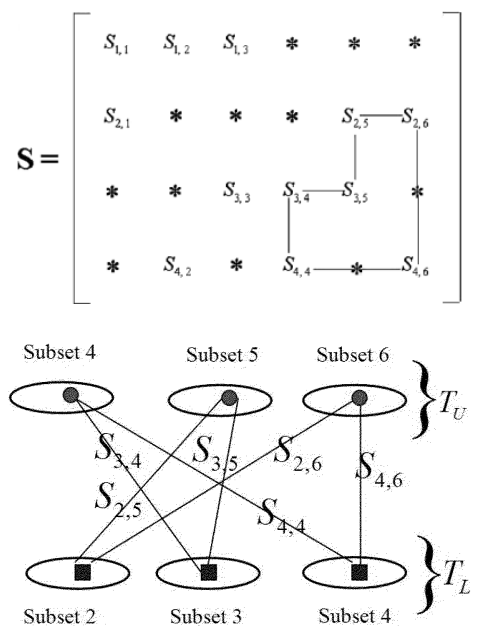

Fig. 2. Top: Closed path in S. Bottom: A 6-cycle in the Tanner graph.

If a $2 t$-cycle exists in the Tanner graph, it can be represented by a vertex sequence $\left\{A_{1}, A_{2}, \ldots, A_{2 t}, A_{1}\right\}$ where the first and the last vertices are the same. Without loss of generality, assume $A_{1}$ is a check node with index $X_{A_{1}}$. Then, by this choice, $A_{2 i-1}, i=1,2, \ldots, t$, are check nodes and $A_{2 i}, i=1,2, \ldots, t$, are bit nodes.

Let $X_{A_{i}}$ represent the index for the vertex $A_{i}, i=$ $1,2, \ldots, 2 t$. By our code construction, there exists shift $S_{\alpha_{2 i-1}, \beta_{2 i-1}}$ and $S_{\alpha_{2 i}, \beta_{2 i}}$ such that

$$
\begin{aligned}
X_{A_{2 i}} & =X_{A_{2 i-1}} \stackrel{p}{\oplus} S_{\alpha_{2 i-1}, \beta_{2 i-1}} i=1, \ldots, t \\
X_{A_{2 i+1}} & =X_{A_{2 i}} \stackrel{p}{\ominus} S_{\alpha_{2 i}, \beta_{2 i}} i=1, \ldots, t-1 .
\end{aligned}
$$

Particularly, we have

$$
X_{A_{1}}=X_{A_{2 t}} \stackrel{p}{\ominus} S_{\alpha_{2 t}, \beta_{2 t}} .
$$

Summing the above $2 t$ equations and cancelling the terms that appear on both sides of the equation, we have

$$
X_{A_{1}}=X_{A_{1}} \stackrel{p}{\oplus}\left(\oplus_{i=1}^{2 t}(-1)^{i+1} S_{\alpha_{i}, \beta_{i}}\right)
$$

hence

$$
\oplus_{i=1}^{2 t}(-1)^{i+1} S_{\alpha_{i}, \beta_{i}}=0 .
$$

In addition, we observe that the $2 t$ shifts $S_{\alpha_{i}, \beta_{i}}, i=1,2, \ldots, 2 t$ associated with the $2 t$-cycle are actually consecutive corners of a closed path of length $2 t$ in the shift matrix $\mathbf{S}$. For example, Fig. 2 on the bottom shows a 6-cycle. Its six associated shifts $S_{2,5}, S_{3,5}, S_{3,4}, S_{4,4}, S_{4,6}$, and $S_{2,6}$ are the six corners of a closed path of length 6 (bold line) in $\mathbf{S}$, as shown on the top in Fig. 2. According to the above observation and (6), we conclude that there must exist a closed path in $\mathbf{S}$ whose $2 t$ corners $S_{\alpha_{1}, \beta_{1}}, S_{\alpha_{2}, \beta_{2}}, \ldots, S_{\alpha_{2 t}, \beta_{2 t}}$ satisfy the Cycle Condition in the Theorem.

only if The only if part can be similarly proved. The details are omitted here. This completes the proof.

\section{GIRTH BOUND}

Theorem 1 presents the Cycle Condition to prevent $2 t$-cycles. However, Theorem 1 does not tell us whether or not we can 
actually find a shift matrix $\mathbf{S}$ where all closed paths of length $2 t$ violate the Cycle Condition. The answer to the above question is addressed in this section in Theorems 2 and 3.

\section{A. Upper Bound on Girth}

Theorem 2: Every PS-LDPC code constructed from a shift matrix $\mathbf{S}$ has girth $g \leq 2\left(L\left(\mathbf{C}_{\mathbf{1}}\right)+L\left(\mathbf{C}_{\mathbf{2}}\right)-L\left(\mathbf{C}_{\mathbf{1}} \cap \mathbf{C}_{\mathbf{2}}\right)\right)$ where $\mathbf{C}_{\mathbf{1}}$ and $\mathbf{C}_{2}$ are any two arbitrary closed paths in $\mathbf{S}$ and $\mathbf{C}_{\mathbf{1}} \cap \mathbf{C}_{\mathbf{2}} \neq \phi$.

Proof: Let $\mathbf{C}_{\mathbf{1}}=\left\{S_{\alpha_{1}, \beta_{1}}, \ldots, S_{\alpha_{i}, \beta_{i}}, S_{\alpha_{i+1}, \beta_{i+1}}\right.$, $\left.\ldots, S_{\alpha_{2 t}, \beta_{2 t}}\right\}$ and $\mathbf{C}_{\mathbf{2}}=\left\{S_{\alpha_{1}, \beta_{1}}, \ldots, S_{\alpha_{i}, \beta_{i}}, S_{\alpha_{i+1}^{\prime}, \beta_{i+1}^{\prime}}, \ldots\right.$, $\left.S_{\alpha_{2 r}^{\prime}, \beta_{2 r}^{\prime}}\right\}$ be two closed paths in $\mathbf{S}$ that share $i$ corners $S_{\alpha_{1}, \beta_{1}}, S_{\alpha_{2}, \beta_{2}}, \ldots, S_{\alpha_{i}, \beta_{i}}$. Consider the closed path represented by the following corner sequence $\mathbf{P}^{*}=$ $\mathbf{P}_{1} \cup \mathbf{P}_{2} \cup \mathbf{P}_{3} \cup \mathbf{P}_{4} \cup \mathbf{P}_{5} \cup \mathbf{P}_{6}$ where the corner sequences $\mathbf{P}_{\mathbf{1}}=\left\{S_{\alpha_{i+1}, \beta_{i+1}}, \ldots, S_{\alpha_{2 t}, \beta_{2 t}}\right\}, \mathbf{P}_{\mathbf{2}}=$ $\left\{S_{\alpha_{1}, \beta_{1}}, \ldots, S_{\alpha_{i}, \beta_{i}}\right\}, \mathbf{P}_{\mathbf{3}}=\left\{S_{\alpha_{i+1}^{\prime}, \beta_{i+1}^{\prime}}, \ldots, S_{\alpha_{2 r}^{\prime}, \beta_{2 r}^{\prime}}\right\}, \mathbf{P}_{\mathbf{4}}=$ $\left\{S_{\alpha_{2 t}, \beta_{2 t}}, \ldots, S_{\alpha_{i+1}, \beta_{i+1}}\right\}, \mathbf{P}_{\mathbf{5}} \stackrel{=}{=}\left\{S_{\alpha_{i}, \beta_{i}}, \ldots, S_{\alpha_{1}, \beta_{1}}\right\}$, and $\mathbf{P}_{6}=\left\{S_{\alpha_{2 r}^{\prime}, \beta_{2 r}^{\prime}}^{\prime} \ldots, S_{\alpha_{i+1}^{\prime}, \beta_{i+1}^{\prime}}\right\}$. The length of $\mathbf{P}^{*}$ is $L\left(\mathbf{P}^{*}\right)=2\left(L\left(\mathbf{C}_{\mathbf{1}}\right)+L\left(\mathbf{C}_{\mathbf{2}}\right)-L\left(\mathbf{C}_{\mathbf{1}} \cap \mathbf{C}_{\mathbf{2}}\right)\right)$.

We compute the summation

$$
\oplus_{i=1}^{L\left(\mathbf{P}^{*}\right)}(-1)^{i+1} S_{\alpha_{i}, \beta_{i}}
$$

for all the corners of $\mathbf{P}^{*}$. We notice that each distinct shift appears exactly twice in (7) with different plus and minus signs. For example, $S_{\alpha_{2 t}, \beta_{2 t}}$ is contained both in $\mathbf{P}_{\mathbf{1}}$ and $\mathbf{P}_{\mathbf{4}}$. When $i$ is even, the $S_{\alpha_{2 t}}, \beta_{2 t}$ contained by $\mathbf{P}_{\mathbf{1}}$ is with the minus sign in (7) and the $S_{\alpha_{2 t}, \beta_{2 t}}$ contained by $\mathbf{P}_{4}$ is with the plus sign in (7). Hence, the summation (7) is always zero regardless of the choice of the shifts in $\mathbf{P}^{*}$, which means the Cycle Condition in Theorem 1 is satisfied for any possible choice of the shifts in $\mathbf{P}^{*}$. By Theorem 1, we derive that there must exist a cycle of length $L\left(\mathbf{P}^{*}\right)$ in the Tanner graph. Therefore, the girth of the Tanner graph is less than or equal to $L\left(\mathbf{P}^{*}\right)=2\left(L\left(\mathbf{C}_{\mathbf{1}}\right)+L\left(\mathbf{C}_{\mathbf{2}}\right)-\right.$ $\left.L\left(\mathbf{C}_{\mathbf{1}} \cap \mathbf{C}_{\mathbf{2}}\right)\right)$. This completes the proof.

\section{B. Achieving the Girth Bound}

We will show next that the upper bound on girth $g$ in Theorem 2 is achievable.

Theorem 3: We can construct PS-LDPC codes with any girth $g \leq 2 \min _{\mathbf{C}_{1}, \mathbf{C}_{2}}\left(L\left(\mathbf{C}_{\mathbf{1}}\right)+L\left(\mathbf{C}_{\mathbf{2}}\right)-L\left(\mathbf{C}_{\mathbf{1}} \cap \mathbf{C}_{\mathbf{2}}\right)\right)$ based on a shift matrix $\mathbf{S}$ where $\mathbf{C}_{\mathbf{1}}$ and $\mathbf{C}_{\mathbf{2}}$ denote two arbitrary closed paths in $\mathbf{S}$ and $\mathbf{C}_{\mathbf{1}} \cap \mathbf{C}_{\mathbf{2}} \neq \phi$.

Proof: The proof is given in Appendix A.

By Theorem 3, we derive the following Corollary.

Corollary 1: We can construct PS-LDPC codes with any desired girth $g_{c} \leq 3 g$ from a small PS-LDPC code with girth $g$.

Proof: Represent the parity-check matrix of the girth $g$ PS-LDPC code by $\mathbf{H}$. Let $\mathbf{H}$ be the incidence matrix of the shift matrix $\mathbf{S}$. Since $\mathbf{H}$ has girth $g$, then every closed path in $\mathbf{S}$ has length at least $g$. Let $\mathbf{C}_{\mathbf{1}}$ and $\mathbf{C}_{\mathbf{2}}$ be two closed paths in $\mathbf{S}$ and $\mathbf{C}_{\mathbf{1}} \cap \mathbf{C}_{\mathbf{2}} \neq \phi$. We have

$$
L\left(\mathbf{C}_{\mathbf{1}}\right) \geq g \text { and } L\left(\mathbf{C}_{\mathbf{2}}\right) \geq g \text {. }
$$

We also notice that $\mathbf{C}_{\mathbf{1}}+\mathbf{C}_{\mathbf{2}}-2 \mathbf{C}_{\mathbf{1}} \cap \mathbf{C}_{\mathbf{2}}$ composes a closed path in $\mathbf{S}$ when $\mathbf{C}_{\mathbf{1}} \cap \mathbf{C}_{\mathbf{2}} \neq \phi$. Hence

$$
L\left(\mathbf{C}_{\mathbf{1}}+\mathbf{C}_{\mathbf{2}}-2 \mathbf{C}_{\mathbf{1}} \cap \mathbf{C}_{\mathbf{2}}\right) \geq g \text {. }
$$

Summing inequalities (8) and (9) together, we have

$$
\begin{aligned}
2\left(L\left(\mathbf{C}_{\mathbf{1}}\right)+L\left(\mathbf{C}_{\mathbf{2}}\right)-L\left(\mathbf{C}_{\mathbf{1}} \cap \mathbf{C}_{\mathbf{2}}\right)\right) & \\
& \geq g+g+g=3 g .
\end{aligned}
$$

By Theorem 3 and inequality (10), we can construct a PS-LDPC code with any girth $g_{c} \leq 3 g$. This completes the proof.

\section{PS-LDPC CODES: ARBITRARY GIRTH}

By Corollary 1, we can translate a difficult problem of designing PS-LDPC codes with girth $3 g$ into a much easier task of designing PS-LDPC codes with girth $g$. If PS-LDPC codes with girth $g$ is still difficult to construct, we can further reduce the girth requirement to $\lceil g / 3\rceil$. Keep decreasing the girth requirement in this way, we will eventually come to the problem of designing PS-LDPC codes with girth $g \leq 12$, By Corollary 1 , PS-LDPC codes with column weight $j$, row weight $k$, and girth $g \leq 12$ can be designed according to a $j \times k$ all " 1 " matrix.

We use Corollary 1 to describe an algorithm that finds a PS-LDPC code with girth $g_{c} \leq 3 g$ based on a PS-LDPC code with girth $g$. Algorithm 1 is as follows:

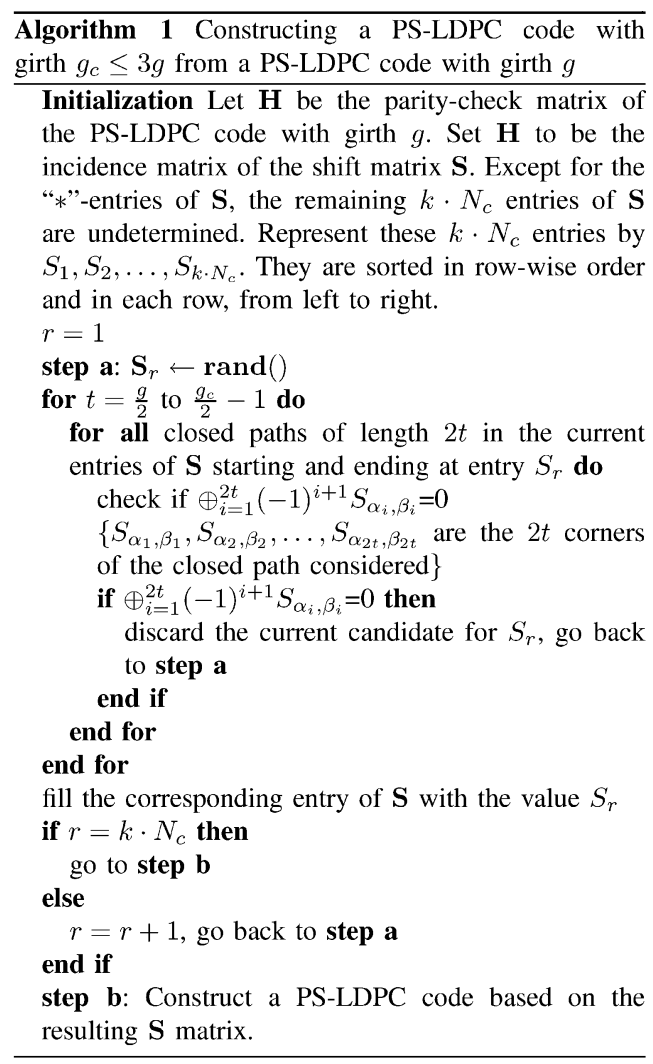

The way we search for shifts in Algorithm 1 is similar to the progressive edge growth (PEG) method [10].

We then propose our main algorithm to construct PS-LDPC codes with arbitrary girth $g$ based on Algorithm 1. Algorithm 2 is as follows: 


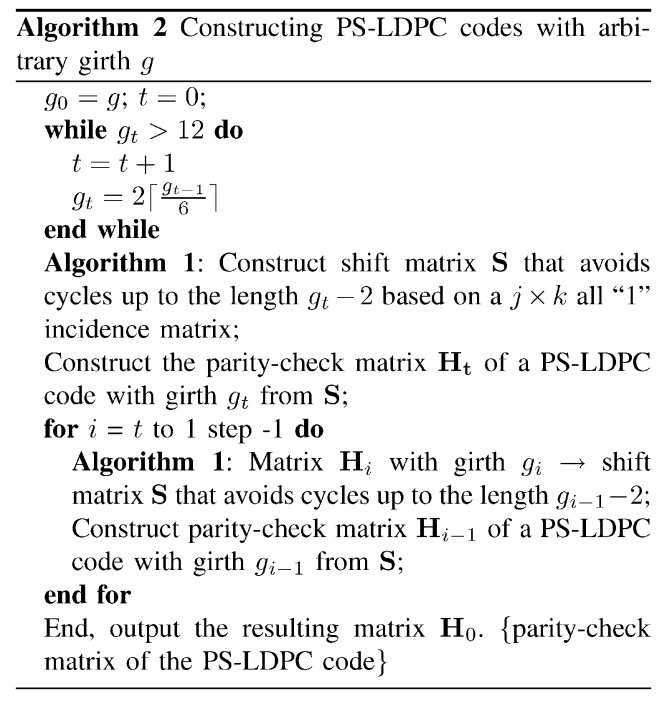

\section{Minimum Distance AnAlysis}

By Corollary 1, large PS-LDPC codes with large girth can be constructed iteratively from smaller codes with small girth. In this way, we can construct PS-LDPC codes with arbitrary large girth $g$.

In addition, Tanner [2] proved the following lower bound on $d_{\min }$ in terms of girth $g$ and bit node degree $j$

$$
\begin{aligned}
& d_{\min } \geq 2\left[(j-1)^{g / 4}-1\right] \div(j-2) \quad g / 2 \text { is even } \\
& d_{\min } \geq\left[j(j-1)^{\left\lfloor\frac{g}{4}\right\rfloor}-2\right] \div(j-2) \quad g / 2 \text { is odd. }
\end{aligned}
$$

Combining Corollary 1 and inequalities (11) and (12) together, we see that PS-LDPC codes can be designed to have arbitrary large minimum distance $d_{\min }$.

\section{PS-LDPC CODES: PERFORMANCE STUdY}

As an illustration, we apply Algorithms 1 and 2 to construct a PS-LDPC code with column weight $j=3$, row weight $k=4$, and girth 14. By Algorithm 2, we need to construct a $3 \times 4 \mathbf{S}$ matrix that avoids 4-cycles first. Using Algorithm 1, we choose the cardinality $p_{1}=5$ and construct the following $3 \times 4$ shift matrix $\mathbf{S}$ that avoids cycles with length 4

$$
\mathbf{S}=\left[\begin{array}{llll}
0 & 0 & 0 & 0 \\
0 & 4 & 1 & 2 \\
0 & 2 & 3 & 1
\end{array}\right]
$$

Based on $\mathbf{S}$, we then construct a $15 \times 20$ parity-check matrix $\mathbf{H}_{\mathbf{1}}$ with girth 6, as shown on the left in Fig. 3.

Next, according to $\mathbf{H}_{\mathbf{1}}$, we build a PS-LDPC code with $j=$ $3, k=4$, cardinality $p_{0}=607$, and girth 14 by Algorithm 1 . The above process results in a $(n=12140, j=3, k=4)$ PS-LDPC code with girth 14 and whose structure is described by the $9105 \times 12140$ matrix $\mathbf{H}_{0}$ shown on the right in Fig. 3 .

We evaluate by simulation the bit error rate (BER) of the PS-LDPC codes. The codes are decoded with the sum-product algorithm [3], and we adopt the signal-to-noise ratio (SNR) defined in [11]: SNR $=10 \log _{10}\left[E_{b} / N_{0}\right]$ where $E_{b}$ denotes
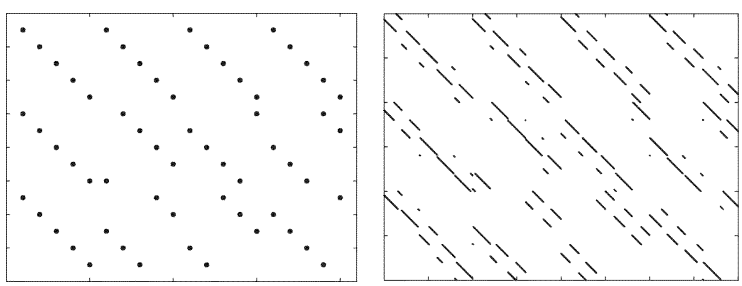

Fig. 3. Left: Parity-check matrix $\mathbf{H}_{\mathbf{1}}$ with girth 6. Right: Parity-check matrix $\mathbf{H}_{\mathbf{0}}$ for a $(12140,3,4)$ PS-LDPC code with girth 14.

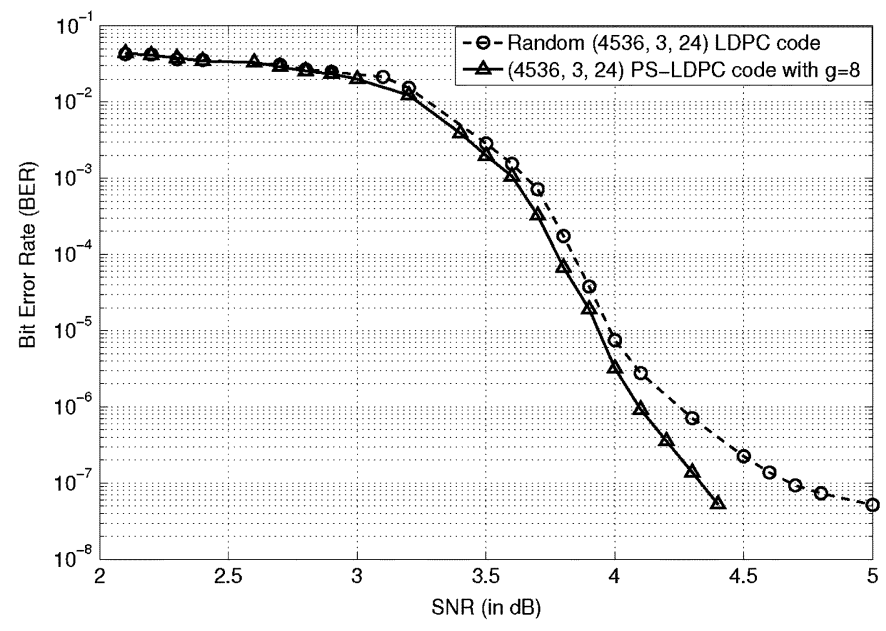

Fig. 4. A PS-LDPC code with girth 8 versus random LDPC code with the same code parameters.

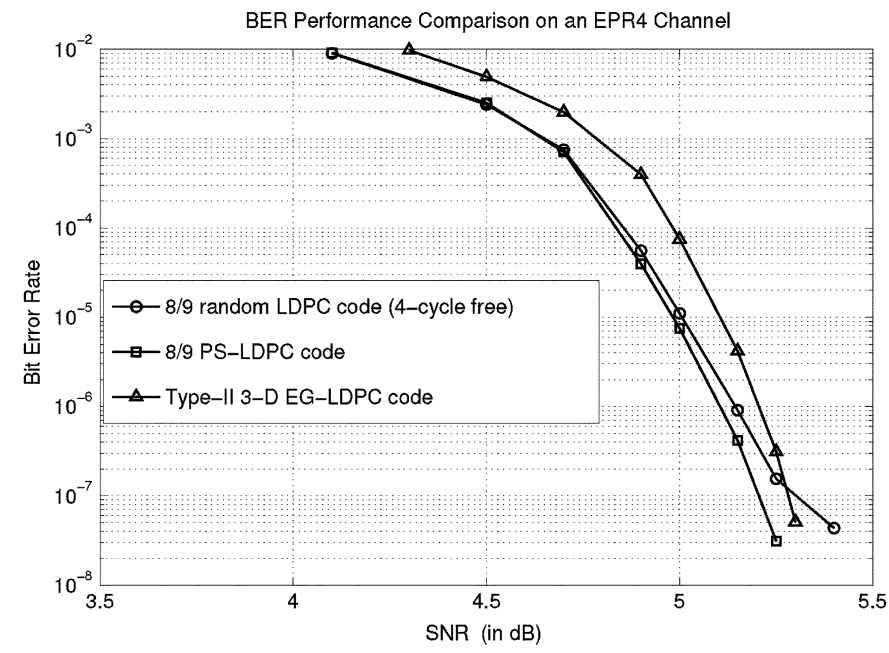

Fig. 5. BER performance comparison I in EPR4 channels.

the user bit energy and $N_{0} / 2$ is the two-sided power spectral density.

The plot in Fig. 4 shows the BER performance for a column weight $j=3$ PS-LDPC code with girth 8 (solid line) in an AWGN channel. For comparison, we also show the BER performance of a randomly constructed LDPC code (no 4-cycles) (dashed line). Both codes have the same block length 4536 and the same code rate $7 / 8$. In the high SNR, the PS-LDPC code outperforms the random LDPC code by SNR $=0.6 \mathrm{~dB}$ at $\mathrm{BER}=5 \times 10^{-8}$ where the performance of the random code has bottomed while the PS-LDPC code has not yet reached the error floor at this BER. 


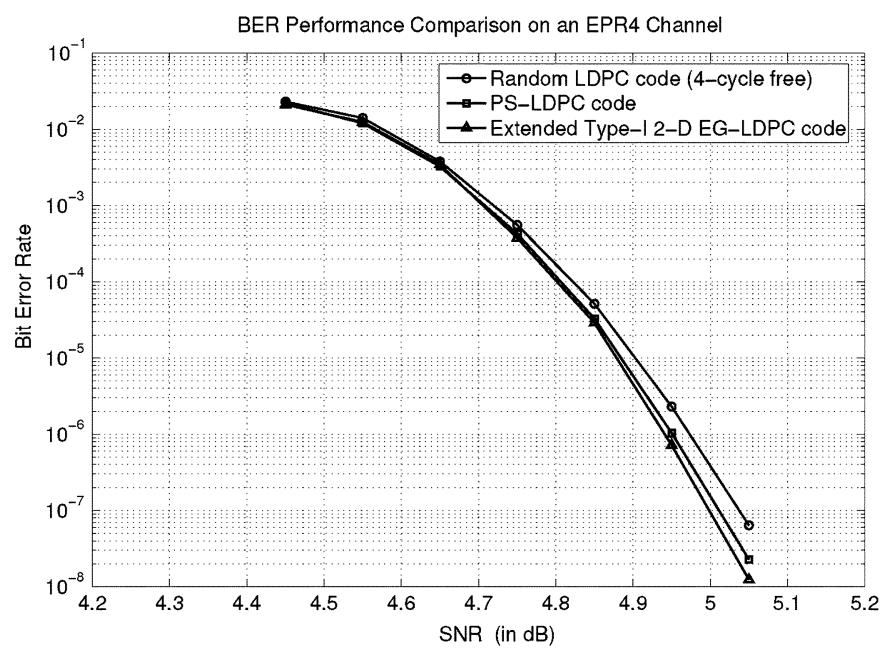

Fig. 6. BER performance comparison II in EPR4 channels.

We also carry on simulations in more realistic channels-EPR4 channels. The BCJR algorithm is used in the channel detector and the reliability information is exchanged between the LDPC decoder and the soft-in-soft-output channel detector to increase the coding gain. The plot in Fig. 5 shows the BER performance for a $(n=6075, j=3, k=27)$ PS-LDPC code with girth 8 in an EPR4 channel. For comparison, we also show the BER performance of a same size random LDPC code (no 4-cycles) and a type-II 3-D finite geometry LDPC code [4] with code length 4599 and code rate 0.92. In the high SNR region, the PS-LDPC code outperforms the random code. More importantly, the slope of the BER curve for the random LDPC code tends to be flat in the high SNR region, implying that for this code the error floor will occur soon, which is not the case for the PS-LDPC code with girth 8. The finite geometry LDPC code shows no error floor as our PS-LDPC code.

The plot in Fig. 6 compares the BER performance for a PS-LDPC code with column weight 4 , code rate $8 / 9$, code length 32760, and girth 8 in an EPR4 channel. We also show the BERs of a $(32760,4,36)$ random LDPC code (no 4-cycles) and an extended type-I 2-D finite geometry LDPC code [4] with code length 32760 and code rate 0.875 . The slope of the BER curve for the PS-LDPC code decreases faster with SNR than that of random LDPC code; PS-LDPC code and the finite geometry LDPC code have similar BER performance.

\section{CONCLUSION}

The paper proposes a new type of regular structured LDPC codes - partition-and-shift LDPC codes. It presents methods to construct PS-LDPC codes with arbitrarily specified girth $g$ and arbitrarily specified degrees $j$ and $k$ for the check nodes and bit nodes, and hence to design flexible code rate PS-LDPC codes. In addition, their parity-check matrix $\mathbf{H}$ is obtained from a much smaller matrix - the shift matrix $\mathbf{S}$, which reduces their storage memory requirements; they also exhibit good BER performance as shown by simulations-a low error floor. All these characteristics make PS-LDPC codes particularly attractive in high-density magnetic recording.

\section{APPENDIX A \\ PROOF OF THEOREM 3}

Define unacceptable values for an entry $S_{\alpha, \beta}$ of the shift matrix $\mathbf{S}$ as follows: $S_{\alpha, \beta}$ is unacceptable if it leads to a cycle of length $g-2$ or smaller. We prove the Theorem by arguing that the number of candidate values for $S_{\alpha, \beta}$ can be made greater than the number of unacceptable values for $S_{\alpha, \beta}$.

By Theorem 1, we first count the number of closed paths with length $2 t$ that start and end at $S_{\alpha, \beta}$. Let the closed path start with a vertical line at $S_{\alpha, \beta}$. There are at most $(j-1)$ entries where this first vertical line can terminate, as there are only $j$ non-“*” entries in the column $\beta$ of $\mathbf{S}$ and the vertical line can not end at the starting corner $S_{\alpha, \beta}$. Any horizontal line from any one of these $(j-1)$ non-“ $*$ " entries can terminate at most at $(k-1)$ entries of $\mathbf{S}$, namely the $k-1$ non-“*” entries in the corresponding row other than the entry where the horizontal line starts. Hence, there are $(j-1)(k-1)$ paths of length 2 that start with a vertical line at $S_{\alpha, \beta}$. Similarly, we derive that there are $\left[(j-1(k-1)]^{t-1}\right.$ paths of length $2 t-2$ that start with a vertical line at $S_{\alpha, \beta}$. Recall that we need to construct a closed path of length $2 t$ ending at $S_{\alpha, \beta}$. Hence, the $(2 t-1)$ th line must terminate at the $\alpha$ th row of $\mathbf{S}$ and the (2t)th line must terminate at the entry $S_{\alpha, \beta}$. Thus, there are $[(j-1)(k-1)]^{t-1}$ closed paths of length $2 t$ that start with a vertical line at $S_{\alpha, \beta}$.

Let $\mathcal{N}\left(S_{\alpha, \beta}\right)$ be the total number of closed paths with length $2 t, 4 \leq 2 t<g$, that pass through the entry $S_{\alpha, \beta}$. Thus, $\mathcal{N}\left(S_{\alpha, \beta}\right)$ is bounded by

$$
\begin{aligned}
\mathcal{N}\left(S_{\alpha, \beta}\right) & \leq \sum_{t=2}^{(g / 2)-1}[(j-1)(k-1)]^{t-1} \\
& <\frac{[(j-1)(k-1)]^{(g / 2-1)}}{j \cdot k-j-k} .
\end{aligned}
$$

By Theorem 1, each of the closed paths with length $2 t, 4 \leq 2 t<$ $g$, is associated with a Cycle Condition $\oplus_{i=1}^{2 t}(-1)^{i+1} S_{\alpha_{i}, \beta_{i}}=$ 0 , which determines an unacceptable value for $S_{\alpha, \beta}$. Therefore, the total number of unacceptable values for $S_{\alpha, \beta}$ is the same as $\mathcal{N}\left(S_{\alpha, \beta}\right)$.

By our code construction, the entry $S_{\alpha, \beta}$ can take $p$ values from 0 to $p-1$. If we let $p \geq[(j-1)(k-1)]^{(g / 2-1)} /(j \cdot k-j-k)>\mathcal{N}\left(S_{\alpha, \beta}\right)$ by inequality (14), the number of unacceptable values for $S_{\alpha, \beta}$ is less than the number of candidate values for $S_{\alpha, \beta}$. Thus, there exist at least one candidate value for $S_{\alpha, \beta}$ that is allowable. Hence, we can construct a shift matrix $\mathbf{S}$ that avoids cycles up to the length $g-2$. This completes the proof.

\section{ACKNOWLEDGMENT}

This research was supported by the Data Storage Systems Center (DSSC) at Carnegie Mellon University.

\section{REFERENCES}

[1] R. G. Gallager, Low-Density Parity Check Codes. Cambridge, MA: MIT Press, 1963.

[2] R. M. Tanner, "A recursive approach to low complexity codes," IEEE Trans. Inf. Theory, vol. IT-27, no. 5, pp. 533-547, Sep. 1981. 
[3] F. R. Kschischang, B. J. Frey, and H. A. Loeliger, "Factor graphs and the sum-product algorithm," IEEE Trans. Inf. Theory, vol. 47, no. 2, pp. 498-519, Feb. 2001.

[4] Y. Kou, S. Lin, and M. P. C. Fossorier, "Low-density parity-check codes based on finite geometries: A rediscovery and new results," IEEE Trans. Inf. Theory, vol. 47, no. 7, pp. 2711-2736, Nov. 2001.

[5] B. Vasic, "Structured iteratively decodable codes based on Steiner systems and their application in magnetic recording," Proc. IEEE Globecom 2001, vol. 5, pp. 2954-2960, Nov. 2001.

[6] H. Tang, J. Xu, Y. Kou, S. Lin, and K. A. S. Abdel-Ghaffar, "On algebraic construction of Gallager and circulant low-density parity-check codes," IEEE Trans. Inf. Theory, vol. 50, no. 6, pp. 1269-1279, Jun. 2004.

[7] M. P. C. Fossorier, "Quasicyclic low-density parity-check codes from circulant permutation matrices," IEEE Trans. Inf. Theory, vol. 50, no. 8, pp. 1788-1793, Aug. 2004.
[8] B. Vasic and O. Milenkovic, "Combinatorial constructions of low-density parity-check codes for iterative decoding," IEEE Trans. Inf. Theory, vol. 50, no. 6, pp. 1156-1176, Jun. 2004.

[9] J. M. F. Moura, J. Lu, and H. Zhang, "Structured LDPC codes with large girth," IEEE Signal Process. Mag., vol. 21, no. 1, pp. 42-55, Jan. 2004.

[10] X. Y. Hu, E. Eleftheriou, and D. M. Arnold, "Regular and irregular progressive edge-growth Tanner graphs," IEEE Trans. Inform. Theory, submitted for publication.

[11] D. J. C. Mackay, "Good error-correcting codes based on very sparse matrices," IEEE Trans. Inf. Theory, vol. 45, no. 2, pp. 399-431, Mar. 1999.

Manuscript received July 1, 2005 (e-mail: tianjin0129@yahoo.com). 